\title{
Bridging Probabilistic and Fuzzy Approaches to Choice Under Uncertainty
}

\author{
Davide Martinetti ${ }^{1}$ Susana Díaz ${ }^{2}$ Susana Montes ${ }^{3}$ Bernard De Baets $^{4}$ \\ ${ }^{1,2,3}$ Dept. of Statistics and Operational Research, University of Oviedo, E-33071 Oviedo, Spain \\ ${ }^{4}$ Dept. of Mathematical Modelling, Statistics and Bioinformatics, Ghent University, B-9000 Ghent, Belgium
}

\begin{abstract}
Imprecise choices can be described using either a probabilistic or a fuzzy formalism. No relation between them has been studied so far. In this contribution we present a connection between the two formalisms that strongly makes use of fuzzy implication operators and t-norms. In this framework, Luce's Choice Axiom turns out to be a special case when the product t-norm is considered and other similar choice axioms can be stated, according to the t-norm in use. Also a new family of operators for transforming bipolar relations into unipolar ones is presented.
\end{abstract}

Keywords: Fuzzy choice function, probabilistic choice function, fuzzy implication operator, fuzzy revealed preference, reciprocal relation, bipolar/unipolar scale.

\section{Introduction}

According to Fishburn [6], there exist at least three ways of representing choices in a mathematical way: binary relations, choice functions or choice probabilities. All of them are legitimate and prove to be appropriate in certain circumstances and the relations between them have been studied in the literature. In particular, the connections between binary relations and choice functions have been analyzed in depth, giving birth to an extensive literature that goes under the name of choice theory with revealed preferences (see $[1,15,16,17,18,19]$ ). Fishburn [6] already addressed the lack of results on the connections between choice functions and probabilistic choice functions and hence he proved a set of propositions on the conditions that should be satisfied by the probabilistic choice function in order for the associated choice function to be rational.

The same situation appears in the framework of fuzzy choice and fuzzy preferences. In the last years the results of classical (crisp) revealed choice theory have been extended to the fuzzy framework, laying bare the connections between fuzzy preference relations and fuzzy choice functions (see, amongst others, $[2,3,7,10,11,12,14,20,21])$. Surprisingly, while the connection between fuzzy preference relations and fuzzy choice functions has been studied in depth, there appears to be no literature on the comparison of fuzzy choice functions and probabilistic choice functions. Recently, we approached this problem and in [13] we proved some preliminary results under the assumption that the t-norm used is the product. An interesting connection between the probabilistic and the fuzzy formalisms was established, in which Luce's Choice Axiom played a key role.

In the present contribution we show new connections between the probabilistic and fuzzy choice formalisms, which are valid for every left-continuous t-norm. We exhibit a set of rationality conditions to be imposed on the probabilistic choice function, which have been inspired by Luce's Axiom of Choice. Furthermore, we propose a new family of operators that can transform a preference relation expressed on a bipolar scale into another one with unipolar behavior.

In order to connect both approaches, we make use of implication operators, trying to maintain the semantics of uncertainty associated to the formalism of probabilistic choice functions and the semantics of fuzziness associated to the fuzzy choice functions.

\section{Preliminary concepts}

This section is devoted to the basic definitions that will be used in the rest of the work: firstly, probabilistic choice functions and reciprocal relations are introduced, then a brief reminder on fuzzy logic connectives and finally the elementary notions on fuzzy choice are presented.

\subsection{Probabilistic choice functions and reciprocal relations}

Let $X$ be a finite set of alternatives and $\mathcal{B}$ be the family of all non-empty subsets of $X\left(\mathcal{B}=2^{X} \backslash\{\varnothing\}\right)$. Imagine that a decision maker is asked to make his choices in the set $X$, when different bundles of alternatives are presented to him. The set $X$ can be a set of products on the shelf at a supermarket. The decision maker is a consumer, whose purchases are recorded. Not all the possible products in $X$ are always available (seasonal products, out-ofstock, etc.). Hence the choices of the decision maker are recorded also considering these restrictions on the available products. A probability measure $P$ on $\mathcal{B}$ can be defined: for every pair $A, B \in \mathcal{B}$, such that $A \subseteq B, P(A, B)$ is the probability that the choice 
from the set $B$ lies in the subset $A$. The probability measure $P$ is completely determined by its values $P(x, A)=P(\{x\}, A)$ (probability of choosing alternative $x$ from the set $A$ ), in the sense that $P(A, B)=\sum_{x \in A} P(x, B)$. This probability measure is also called probabilistic choice function and associates to every $(x, A)$ in $X \times \mathcal{B}$ a value $P(x, A)$ that represents the probability that element $x$ is chosen, when $A$ is available. The function $P$ can be approximated using the frequency of observations in a dataset. For convenience, we will denote the choice over the pairs by $p(x, y)=P(\{x\},\{x, y\})$. This relation $p$ is usually called probabilistic relation or reciprocal relation in the literature and obviously satisfies the condition $p(x, y)+p(y, x)=1$, for any $x, y$ in $X$. We understand that the value of $p(x, y)$ represents the preference of $x$ over $y$ in a pairwise comparison. One interesting aspect of reciprocal relations is that they show a bipolar semantics, in the sense that depending on the value taken by $p(x, y)$ in the unit interval, we can talk about two different situations:

i) if $p(x, y) \in\left[\frac{1}{2}, 1\right]$, then $p$ is expressing preference of alternative $x$ over $y$,

ii) if $p(x, y) \in\left[0, \frac{1}{2}\right]$, then $p$ is expressing preference of alternative $y$ over $x$.

Obviously, if $p(x, y)=\frac{1}{2}$, then we consider $x$ and $y$ as indifferent. One advantage of the bipolar scale is that the knowledge of the value $p(x, y)$ is sufficient for understanding the relation standing between the alternatives $x$ and $y$.

\subsection{Binary operations}

When working in the fuzzy framework it is essential to define different operators that play the role of classical Boolean operators. Triangular norms are widely used in this sense and their definition and properties are summarized in this section. We will use the notation $\wedge$ and $\vee$ for the minimum and maximum operators, respectively.

Definition 1 A triangular norm (t-norm for short) is a binary operation * on $[0,1]$ such that for any $a, b, c \in[0,1]$ the following properties are verified:
i) commutativity: $a * b=b * a$;
ii) associativity: $a *(b * c)=(a * b) * c$;
iii) monotonicity: if $a \leq b$, then $a * c \leq b * c$;
iv) neutral element 1: $a * 1=a$.

The three most important t-norms are the minimum, $a *_{M} b=a \wedge b$, the product, $a *_{P} b=a \cdot b$ and the Eukasiewicz operator, $a *_{L} b=(a+b-1) \vee 0$. A t-norm is continuous if it is a continuous two-place function. A t-norm is called left-continuous if all partial mappings are left-continuous.
To any t-norm * we can associate another binary operator called residuum or implication, denoted $\rightarrow_{*}$, defined by:

$$
a \rightarrow_{*} b=\sup \{c \in[0,1] \mid a * c \leq b\},
$$

for any $a, b \in[0,1]$. Given a t-norm * and its residuum operator $\rightarrow_{*}$, another operator called biresiduum can be defined: $a \leftrightarrow_{*} b=\left(a \rightarrow_{*} b\right) \wedge$ $\left(b \rightarrow_{\star} a\right)$. The biresiduum operator between $a$ and $b$ is often interpreted as a measure of how equal $a$ and $b$ are. It turns out that $a \leftrightarrow_{\star} b=1$ if and only if $a=b$.

In Table 1 we list some well-known t-norms and associated residuum and biresiduum operators.

\begin{tabular}{c|c|c}
$a * b$ & $a \rightarrow b$ & $a \leftrightarrow b$ \\
\hline \hline$a *_{M} b$ & $\begin{cases}1 & \text { if } a \leq b, \\
b & \text { else }\end{cases}$ & $\left\{\begin{array}{l}1 \text { if } a=b, \\
a \wedge b \text { else }\end{array}\right.$ \\
\hline$a{ }_{P} b$ & $\begin{cases}1 & \text { if } a \leq b, \\
b / a & \text { else }\end{cases}$ & $\frac{a \wedge b}{a \vee b}$ \\
\hline$a{ }_{L} b$ & $\begin{cases}1 & \text { if } a \leq b, \\
1-a+b & \text { else }\end{cases}$ & $1-|a-b|$
\end{tabular}

Table 1: Three well-known t-norms and associated residuum and biresiduum operators

In this work we are supposing that the t-norm is chosen first and then residuum and biresiduum operators are derived from it according to the previous definitions. In no case we will work with one t-norm $*_{1}$ and a residuum operator derived from another tnorm $\star_{2}$. For this reason, we can avoid the usual notation of residuum and biresiduum operators $\left(\rightarrow_{*}\right.$ and $\leftrightarrow_{*}$ ) in which the dependence on the t-norm has to be made explicit.

We finally recall a short list of properties of leftcontinuous t-norms and their associated residua:

$$
\begin{array}{ll}
\text { Property 1: } & a * b \leq c \Leftrightarrow a \leq b \rightarrow c ; \\
\text { Property 2: } & a \leq b \Leftrightarrow a \rightarrow b=1 ; \\
\text { Property 3: } & 1 \rightarrow a=a ;
\end{array}
$$

\subsection{Fuzzy choice functions and fuzzy preference relations}

A fuzzy choice function $C$ in the sense of Banerjee (see [2]) is defined over $X \times \mathcal{B}$ and associates to every $(x, A)$ a value $C(x, A)$ in the unit interval that represents the degree of preference of alternative $x$ when the set of alternatives $A$ is available. The only condition imposed on $C$ is that, for every $A \in \mathcal{B}$, there exists at least one alternative $x \in A$ such that $C(x, A)>0$. The value of the membership function $C(x, A)$ is interpreted as a numerical encoding of the preference of alternative $x$ among a set of feasible alternatives $A$. There exist other possible interpretations for the membership function $C(\cdot, A)$, 
as pointed out in [5]. The most tempting is the interpretation of $C(x, A)$ as the possibility of $x$ being the alternative chosen from the set $A$.

The fuzzy choice function $C$ satisfies condition $\mathrm{H} 1$ if, for every $A \in \mathcal{B}$, there exists one element $x \in A$, such that $C(x, A)=1$, i.e. the fuzzy set $C(\cdot, A)$ is a normal fuzzy set. It satisfies condition $\mathrm{H} 2$ if the family $\mathcal{B}$ of subsets of $X$ is equal to $2^{X} \backslash\{\varnothing\}$. Condition H2 may sound too demanding, specially in a practical context, where such a complete information is seldom available. In fact, the value of $C(\cdot, A)$ may be known for few sets A's only. Nevertheless, we will show in Section 3 that condition H2 can be satisfied by artificially filling the information on the missing sets, starting from the knowledge on the available ones.

A fuzzy preference relation on $X$ is a mapping $Q: X^{2} \rightarrow[0,1]$ which associates to every pair of elements $x$ and $y$ of $X$ a real value $Q(x, y) \in[0,1]$ that represents the degree of preference of the first element over the second. In this work we consider the following properties of $Q$ :

(i) reflexivity: $Q(x, x)=1$, for every $x \in X$;

(ii) strong completeness: $Q(x, y) \vee Q(y, x)=1$, for every $x, y \in X$;

Unlike reciprocal relations, fuzzy preference relations exhibit an unimodal semantics, in the sense that the value of $R(x, y)$ only represents the preference of alternative $x$ over $y$, giving no information on the opposite relation. For a complete knowledge of the relation standing between $x$ and $y$, both $R(x, y)$ and $R(y, x)$ need to be known.

Definition 2 [2] A fuzzy preference relation $R$ can be revealed from a fuzzy choice function $C$ using the following expression:

$$
R(x, y)=\bigvee_{\{S \in \mathcal{B} \mid x, y \in S\}} C(x, S) .
$$

Definition 3 [2] A fuzzy choice function $C$ is called $G$-rational if there exists a fuzzy preference relation $Q$ such that:

$$
C(x, S)=G(S, Q)(x)=\bigwedge_{y \in S} Q(x, y) .
$$

The capital letter $G$ stands for greatest, since the function $G(S, Q)$ is the fuzzy counterpart of the set of greatest elements used in crisp choice theory.

Definition 4 A fuzzy choice function is called $G$ normal if it is the G-rationalization of a fuzzy preference relation $Q$ and its fuzzy revealed preference relation $R$ is equal to $Q$.

We recall here a result on fuzzy choice functions and revealed preferences:

Proposition 1 [7] If $H 1$ and H2 are verified by a fuzzy choice function $C$, then the fuzzy revealed preference relation $R$ from $C$ is strongly complete and reflexive.

\section{From probabilistic to fuzzy choice functions}

This section contains the main results of the contribution. After presenting our new set of axioms, a transformation from probabilistic choice to fuzzy choice is presented. Special concern is devoted to the transformation of reciprocal relation into fuzzy preference relation too. Theorem 1 concludes the section.

\subsection{A new family of axioms}

In probability theory, Luce's Choice Axiom, formulated by R. Duncan Luce [8, 9], states that the probability of selecting one alternative over another from a set of available alternatives is not affected by the presence or absence of other items in the set. Selection of this kind is said to be independent from irrelevant alternatives (IIA). Formally, it is composed of two parts:

Part 1 If $p(x, y) \neq 0$, for all $x, y$ in $X, x \neq y$, then for all $S \in \mathcal{B}$ and all $x$ in $S$

$$
P(x, X)=P(x, S) P(S, X) .
$$

Part 2 If $p(x, y)=0$, for some $x, y$ in $X$, then for all $S \in \mathcal{B}$, we have

$$
P(S, X)=P(S \backslash\{x\}, X \backslash\{x\}) .
$$

One consequence of Luce's Choice Axiom is that the probabilistic choice function $P$ satisfies the socalled constant ratio rule: for any $x, y$ in $X$, it holds that

$$
\frac{P(x, S)}{P(y, S)}=\frac{P(x, X)}{P(y, X)} .
$$

This condition trivially implies that

$$
\frac{p(x, y)}{p(y, x)}=\frac{P(x, S)}{P(y, S)}, \text { for any } S \in \mathcal{B}
$$

and it has been extensively used in [13] for the study of the connections between probabilistic and fuzzy choice functions, in the case the t-norm is the product. Inspired by its multiplicative nature and the coincidence that it was working well with the product t-norm, we try to generalize this condition to other t-norms. For this reason, we propose the following set of axioms:

Definition 5 Let * be a left-continuous t-norm, $\leftrightarrow$ its associated biresiduum operator and $P$ a probabilistic choice function on $X$. For any $S \subseteq T \in \mathcal{B}$ and $x, y \in S, P$ satisfies

Condition $1 P(x, S) \geq P(y, S)$ implies $P(x, T) \geq$ $P(y, T)$;

Condition $2 p(x, y) \leftrightarrow p(y, x)=P(x, S) \leftrightarrow$ $P(y, S)$. 
The first condition says that if $x$ is preferred to $y$ in $S$, then the same preference should persist when more alternatives are added to the set $S$. It is independent from the choice of the t-norm. The second condition says something more: it establishes that also the degree to which $p(x, y)$ and $p(y, x)$ are similar should be constant when considering a bigger set $S$ that contains both $x$ and $y$. We can say that the first condition concerns the ordering between the alternatives induced by the probabilistic choice function, while the second imposes numerical constraints on the value of the choice function.

Back to the constant ratio rule, it turns out that Eq. (8) is exactly Condition 2 when the t-norm is the product. If we turn to other t-norms, then Conditions 1 and 2 take the following form:

i) if $*$ is the minimum t-norm, then they express that if $p(x, y)>p(y, x)$, then $P(y, S)=p(y, x)$, for any $S \in \mathcal{B}$ such that $x, y \in S$;

ii) if $*$ is the Eukasiewicz t-norm, then they express that the difference between $P(x, S)$ and $P(y, S)$ has to be equal to the difference between $p(x, y)$ and $p(y, x)$, for any $S \in \mathcal{B}$ such that $x, y \in S$.

Conditions 1 and 2 can also be used in case of incomplete knowledge. Suppose that the probabilistic choice function is known only for some subsets of $X$ : conditions 1 and 2 can be used to compute the value of the probabilistic choice function for the missing subsets of $X$, according to some rational conditions.

\subsection{Constructing a fuzzy choice function from a probabilistic choice function}

Following the ideas in [13], we consider the case of a probabilistic choice function $P$ defined over a finite set of alternatives $X$ and we want to construct a fuzzy choice function from it, representing the uncertainty of the probabilistic choice function with the gradualness of a fuzzy choice function. To do this, consider a left-continuous t-norm * and its associated residuum and biresiduum operators. The following equation

$$
\rho(x, S)=\bigwedge_{y \in S} P(y, S) \rightarrow P(x, S)
$$

represents a way of computing a fuzzy choice function $\rho: X \times \mathcal{B} \longrightarrow[0,1]$, for every $x \in X$ and $S \in \mathcal{B}$. Furthermore, if $\mathcal{B}=2^{X} \backslash\{\varnothing\}$, then $\rho$ satisfies condition H2. Also, since in every $S \in \mathcal{B}$ there exists at least one element $x$ such that $P(x, S) \geq P(y, S)$, for any $y \in S$, it immediately follows that $\rho(x, S)=1$ and hence condition $\mathrm{H} 1$ is also satisfied.

A fuzzy preference relation $R$ can be revealed from $\rho$ using Definition 2:

$$
\begin{aligned}
R(x, y) & =\bigvee_{\{S \in \mathcal{B} \mid x, y \in S\}} \rho(x, S) \\
& =\bigvee_{\{S \in \mathcal{B} \mid x, y \in S\}}\left(\bigwedge_{z \in S} P(z, S) \rightarrow P(x, S)\right)
\end{aligned}
$$

and by Proposition 1 we can ensure that $R$ is reflexive and strongly complete.

Proposition 2 If Conditions 1 and 2 are satisfied by a probabilistic choice function $P$ for a given $t$ norm *, then Eq. (10) can be written as a function of the reciprocal relation $p$ :

$$
R(x, y)=p(y, x) \rightarrow p(x, y) .
$$

Proof: Consider an arbitrary pair of different alternatives $x$ and $y$. $\operatorname{Eq}(.10)$ can be written as:

$$
R(x, y)=p(y, x) \rightarrow p(x, y) \vee \underset{\{S \in \mathcal{B}|x, y \in S,| S \mid \geq 3\}}{\bigvee} \rho(x, S)
$$

If $p(y, x) \rightarrow p(x, y)=1$, then $R(x, y)=p(y, x) \rightarrow p(x, y)$. If $p(y, x) \rightarrow p(x, y)<1$, then $p(y, x)>p(x, y)$ and by Condition $1, P(y, S) \geq P(x, S)$, for every $S$ that contains both $x$ and $y$. Consider the second part of Eq. (12): $\bigvee_{\{S \in \mathcal{B}|x, y \in S,| S \mid \geq 3\}} \rho(x, S)$. From conditions 1 and 2 and the definition of the fuzzy choice function $\rho$, we have:

$$
\begin{aligned}
& \bigvee_{\{S \in \mathcal{B}|x, y \in S,| S \mid \geq 3\}} \rho(x, S) \\
= & \bigvee_{\{S \in \mathcal{B}|x, y \in S,| S \mid \geq 3\}}\left(\bigwedge_{z \in S} P(z, S) \rightarrow P(x, S)\right) \\
\leq & \bigvee_{\{S \in \mathcal{B}|x, y \in S,| S \mid \geq 3\}} P(y, S) \rightarrow P(x, S) \\
= & \bigvee_{\{S \in \mathcal{B}|x, y \in S,| S \mid \geq 3\}} P(y, S) \leftrightarrow P(x, S) \\
= & p(y, x) \leftrightarrow p(x, y) \\
= & p(y, x) \rightarrow p(x, y) .
\end{aligned}
$$

Hence the supremum in Eq. (12) is reached always by $p(y, x) \rightarrow p(x, y)$, proving the proposition.

Remark 1 In [4] is presented another transformation of the probability distribution $P$. In that case, the probability distribution is transformed into a possibility distribution, that, as already pointed before, is just another possible interpretation of the fuzzy choice function $C$. The main difference between the transformations presented in Eq. 9 and in Proposition 2 with respect to the one contained in [4] is that ours make strong use of implication operators and the numerical result depends on the initial choice of the t-norm.

On the other hand, it would be nice to write the reciprocal relation $p$ as a function of the fuzzy revealed preference $R$, i.e. $p(x, y)=F(R(x, y), R(y, x))$, for some function $F:[0,1]^{2} \longrightarrow[0,1]$. For example, in $[13]$ we proved that if the t-norm is the product, then $p$ can be computed as follows:

$$
p(x, y)=\frac{R(x, y)}{R(x, y)+R(y, x)} .
$$


Other forms for $F$ can easily be found for the minimum and Łukasiewicz t-norm, while it can be expected that there exists no general form for $F$, since it strongly depends on the t-norm in use. Nevertheless, it can be proved that, provided the t-norm is left-continuous and given $R(x, y)$ and $R(y, x)$, there exists a unique pair $p(x, y)$ and $p(y, x)$, such that

i) $p(x, y)+p(y, x)=1$;

ii) $R(x, y)=p(y, x) \rightarrow p(x, y)$;

iii) $R(y, x)=p(x, y) \rightarrow p(y, x)$.

due to the monotonicity of the t-norm. Hence, even if the function $F$ can not be expressed explicitly, we can ensure that it exists and that it is unique.

The result of Proposition 2 is also interesting from the point of view of the comparison of preference relations with bipolar and unipolar semantics. In fact, Eq. (11) gives a way of expressing a unipolar preference relation $(R)$ as a function of a bipolar one $(p)$. Other works by the same authors already treated this aspect, focusing specifically on the transferability of transitivity properties from one relation to the other. The expression contained in Eq. (11) corresponds to a new family of operators for translating bipolar relations into unipolar relations.

One of the most important properties that a fuzzy choice function can satisfy is G-normality, as proved in $[7,12]$. In fact, a version of the Arrow-Sen Theorem can be proved for fuzzy choice functions, where G-normality plays a crucial role. It would be interesting to find some condition for the probabilistic choice function $P$ that can ensure the G-normality of the derived fuzzy choice function $\rho$. Recall that, according to Definition 4, a fuzzy choice function $\rho$ is G-normal if the G-rationalization of its fuzzy revealed preference $R$ coincides with $\rho$ itself.

Theorem 1 If Conditions 1 and 2 are satisfied by $P$ for some left-continuous t-norm *, then the fuzzy choice function $\rho$ is G-normal.

Proof: The expression given by Eq. (5) is used to compute the G-rationalization of the fuzzy revealed preference $R$, that, taking into account the result of Proposition 2, becomes:

$$
G(S, R)(x)=\bigwedge_{y \in S} R(x, y)=\bigwedge_{y \in S} p(y, x) \rightarrow p(x, y) .
$$

We want to prove that $G(S, R)(x)$ coincides with $\rho(x, S)$, for every $x \in X$ and $S \in \mathcal{B}$. Consider an arbitrary $x \in X$ and $S \in \mathcal{B}$.

If the alternative $x$ is such that $p(x, y) \geq p(y, x)$, for all $y \in S$, then $G(S, R)(x)=1$ and, by Condition 1 , we also know that $P(x, S) \geq P(y, S)$, for every $y \in$ $S$, and hence $\rho(x, S)=\bigwedge_{y \in S} P(y, S) \rightarrow P(x, S)=1$. We can conclude that $G(S, R)(x)=\rho(x, S)$.

If there exist alternatives $\left\{y_{1}, \ldots, y_{n}\right\}$ in $S$ such that $p\left(y_{i}, x\right)>p\left(x, y_{i}\right)$, then let $y_{0}$ be the alternative such that $p\left(y_{0}, x\right) \rightarrow p\left(x, y_{0}\right)$ is the smallest. Then $G(S, R)(x)=p\left(y_{0}, x\right) \rightarrow p\left(x, y_{0}\right)<1$. Due to Condition 1 , we have that $P\left(y_{i}, S\right) \geq P(x, S)$ for all $i \in\{1, \ldots, n\}$ and due to Condition 2 and the definition of the biresiduum operator we also have that:

$$
\begin{aligned}
p\left(y_{i}, x\right) \rightarrow p\left(x, y_{i}\right) & =p\left(y_{i}, x\right) \leftrightarrow p\left(x, y_{i}\right) \\
& =P\left(y_{i}, S\right) \leftrightarrow P(x, S) \\
& =P\left(y_{i}, S\right) \rightarrow P(x, S)
\end{aligned}
$$

Substituting the values of Eq. (15) in the formulas for computing $G(S, R)(x)$ and $\rho(x, S)$, we can conclude that $G(S, R)(x)=p\left(y_{0}, x\right) \rightarrow p\left(x, y_{0}\right)=$ $P\left(y_{0}, S\right) \rightarrow P(x, S)=\rho(x, S)$.

\section{Conclusions and future work}

In this work we proposed a novel way of connecting two important representations of choice when uncertainty is involved: probabilistic choice functions and fuzzy choice functions. Being aware of the semantic differences between the probabilistic and fuzzy approaches, we have been able to define a clear way for passing from one to another, which strongly involves the use of fuzzy implication operators derived from left-continuous t-norms. This connection was still missing in the vast literature on fuzzy representations of choice. A new set of conditions, inspired by Luce's Axiom of Choice, is presented and, depending on the t-norm in use, it can take different forms. Imposing these conditions on the probabilistic choice function ensures that the fuzzy choice function generated from it is G-normal, a property that has been proved to be fundamental in the study of rationality conditions in the fuzzy choice framework. Furthermore, we found new ways of transforming a reciprocal relation into a fuzzy preference relation, making a little step forward in the understanding of the translation of bipolar scales into unipolar ones.

As pointed out in the contribution, the conditions imposed on the probabilistic choice functions depend on the t-norm used and perhaps an ordering can be found, respecting the natural order between t-norms, in such a way that a ranking of the sets of axioms can be established.

\section{Acknowledgments}

The research reported on in this contribution has been partially supported by Project MTM201017844 and the Foundation for the promotion in Asturias of the scientific and technologic research grant BP10-090.

\section{References}

[1] K. J. Arrow, Rational choice functions and orderings, Economica, 26:121-127, 1959. 
[2] A. Banerjee, Fuzzy choice functions, revealed preference and rationality, Fuzzy Sets and Systems, 70:31-43, 1995.

[3] R. Barrett, P. K. Pattanaik and M. Salles, On choosing rationally when preferences are fuzzy, Fuzzy Sets and Systems, 34:197-212, 1990.

[4] D. Dubois, Possibility theory and statistical reasoning, Computational Statistics and Data Analysis, 51:47-69, 2006.

[5] D. Dubois, The role of fuzzy sets in decision sciences: Old techniques and new directions. Fuzzy Sets and Systems, 184:3-28, 2011.

[6] P. C. Fishburn, Choice probabilities and choice functions, Journal of Mathematical Psychology, 18:205-219, 1978.

[7] I. Georgescu, Fuzzy Choice Functions, A revealed Preference Approach, Springer, Berlin, 2007.

[8] R. D. Luce, Individual Choice Behavior: A Theoretical Analysis, Willey, New York, 1959.

[9] R. D. Luce and P. Suppes, Preferences utility and subject probability. In: R. D. Luce, R. R. Bush and E. Galanter, editors, Handbook of Mathematical Psychology III, Wiley, 249-410, 1965.

[10] D. Martinetti, B. De Baets, S. Díaz and S. Montes, On the role of acyclicity in the study of rationality of fuzzy choice functions. In proceedings of $11^{\text {th }}$ International Conference on Intelligent Systems Design and Applications, (ISDA 2011) 350-355, 2011.

[11] D. Martinetti, I. Montes and S. Díaz, From preference relations to fuzzy choice functions. In proceedings of $11^{t} h$ European Conference on Symbolic and Quantitative Approaches to Reasoning with Uncertainty. (ECSQARU 2011), Lecture Notes in Computer Science 6717, 594605, 2011.

[12] D. Martinetti, S. Montes, S. Díaz and B. De Baets, Some comments to the fuzzy version of the Arrow-Sen theorem. In proceedings of $14^{t} h$ International Conference on Information Processing and Management of Uncertainty in Knowledge-Based Systems. (IPMU 2012), Communications in Computer and Information Science 300, 286-295, 2012.

[13] D. Martinetti, S. Montes, S. Díaz and B. De Baets, Uncertain choices: a comparison of fuzzy and probabilistic approaches. To be presented at AGOP 2013.

[14] G. Richardson, The structure of fuzzy preference: Social choice implications, Social Choice and Welfare, 15:359-369, 1998.

[15] M. K. Richter, Revealed preference theory, Econometrica, 34:635-645, 1996.

[16] P. A. Samuelson. Foundation of Economic Analysis, 604 pages, Harvard University Press, Cambridge, Massachusetts, 1983.

[17] A. K. Sen, Choice functions and revealed preference, Review of Economic Studies, 38:307-
317, 1971.

[18] K. Suzumura, Rational choice and revealed preference, Review of Economic Studies, 46:149-158, 1976.

[19] I. Uzawa. Preference and Rational Choice in the Theory of Consumption, Mathematical Methods in the Social Sciences, Arrow, K. J., Karlin, S. and Suppes, P.(eds.), Stanford University Press, 1959.

[20] X. Wang, A note on congruence conditions of fuzzy choice functions, Fuzzy Sets and Systems, 145:355-358, 2004.

[21] X. Wang, C. Wu, and X. Wu, Choice Functions in Fuzzy Environment: An Overview. In Studies in Fuzziness and Soft Computing, vol. 261, 35 Years of Fuzzy Set Theory, C. Cornelis, G. Deschrijver, M. Nachtegael, S. Schockaert and Y. Shi editors, Springer-Verlag Berlin Heidelberg, 149-170, 2010. 